\title{
High incidence of acute self-reported sleep disturbances in patients following arthroscopic- assisted knee surgery
}

\author{
Nicholas N DePhillipo (1) , ${ }^{1,2}$ Robert S Dean, ${ }^{1}$ Lars Engebretsen, ${ }^{3}$ \\ Christopher M Larson, ${ }^{1}$ Jill Monson 지, ${ }^{1}$ Robert F LaPrade ${ }^{1}$
}

${ }^{1}$ Twin Cities Orthopedics, EdinaCrosstown, Edina, Minnesota, USA

${ }^{2}$ Department of Sports Medicine, Oslo Sports Trauma Research Center, Norwegian School of Sport Sciences, Oslo, Norway ${ }^{3}$ Department of Orthopaedic Surgery, Oslo University Hospital, Oslo, Norway

Correspondence to Professor Nicholas N DePhillipo, Twin Cities Orthopedics, Edina, Minnesota, USA;

Nickd.atc@gmail.com

Accepted 21 April 2021 Published Online First 16 July 2021

Check for updates

(C) International Society of Arthroscopy, Knee Surgery and Orthopaedic Sports Medicine 2021. No commercial re-use. See rights and permissions. Published by BMJ.

To cite: DePhillipo NN, Dean RS, Engebretsen $L$, et al. J ISAKOS 2021:6:259-264.

\section{ABSTRACT}

Objectives To evaluate the self-reported incidence of sleep disturbances, defined as $\leq 7$ hours of sleep per 24 hour period, in patients undergoing arthroscopic-assisted knee surgery.

Methods Patients who underwent arthroscopic knee surgery over the course of a 4-month period were prospectively included. Patients were excluded if a history of insomnia or other sleep altering medical history was reported. Self-reported sleep metrics included average number of hours of sleep per night, average number of awakenings during sleep per night, perceived quality of sleep, average pain level during sleep and number of hours of physical activity/therapy per week. Data were collected at weeks 1, 3, and 6 postoperatively. Joint circumference was measured on postoperative day 1 and served as an indicator of a knee effusion. Paired $t$-tests were used to compare preoperative to postoperative hours of sleep. Simple and multiple linear regression were used to evaluate relationships between surgical variables and postoperative sleep metrics.

Results There were 123 patients who underwent arthroscopic knee surgery during the prospective enrolment period; 83 patients were included in the final analysis. The overall incidence of preoperative sleep disturbances was $20 \%(n=17)$. The overall incidence of self-reported postoperative sleep disturbances was $99 \%, 96 \%$ and $90 \%$ at weeks 1,3 and 6 , respectively. The average number of hours slept was significantly reduced at 1, 3 and 6 weeks postoperatively compared with the preinjury state $(p<0.001)$. Knee joint circumference had a significantly negative correlation with average number of hours of sleep in the first 6 weeks postoperatively $(R=-0.704 ; p=0.001)$. Surgical variables including severity of surgery, weekly postoperative pain level and weekly hours of postoperative physical therapy were not significant independent predictors of acute postoperative sleep disturbances ( $p>0.05$ )

Conclusion Sleep disturbances were commonly reported in patients following arthroscopic knee surgery without correction of sleep metrics by 6 weeks postoperatively. The majority of sleep disturbances in this cohort correlated with an increased knee effusion. A multidisciplinary team approach is recommended to counsel patients regarding the potential for and problems with acute sleep disturbances following arthroscopic knee surgery.

Level of evidence: 3.
What are the new findings

- High incidence (>90\%) of acute self-reported sleep disturbances (8.0 hours compared with 5.9 hours, preoperative to postoperative, respectively) following arthroscopic knee surgery.

- Increased knee effusion was significantly correlated with postoperative sleep disturbances.

- Severity of surgery, pain level and hours of physical therapy were not significantly correlated with postoperative sleep disturbances.

- Most commonly reported causes of postoperative sleep disturbances following knee surgery were increased knee temperature/ warmth from knee effusion (96\%), wearing a brace to sleep (90\%) and not sleeping in normal body position compared with presurgery $(87 \%)$.

\section{INTRODUCTION}

Sleep disturbances are commonly reported following musculoskeletal injury/surgery and neurocognitive impairments (eg, concussions). Pain at night and sleep disturbances are often associated with joint osteoarthritis and shoulder pathology such as rotator cuff tears, and previous studies have demonstrated a corrective effect of sleep with recovery after a rotator cuff repair. ${ }^{1-3}$ Substantial evidence suggests that sufficient quantity and quality of sleep are necessary for maintaining normal bodily function, including restoration of damaged tissues and flushing toxins from the brain, while inadequate sleep can have serious long-term health consequences. ${ }^{4-8}$

Not having sufficient sleep has been reported as a public health risk contributing to obesity, cardiovascular disease and mental health disorders. ${ }^{9-11}$ Research has shown that individuals who report short sleep durations (ie, $\leq 7$ hours per night) have a 10\%-30\% increased risk of developing cardiovascular disease. ${ }^{912}$ Insufficient sleep may also trigger the release of various proteins that cause systemic inflammation and cause dysregulation of the immune system. ${ }^{1314}$ These increased inflammatory markers may adversely prolong recovery following orthopaedic-related surgery and/or musculoskeletal injury. ${ }^{13} 15$

Previous data have shown that sleep disturbances are reported in patients following hip and knee 
joint arthroplasty. ${ }^{16} 17$ A recent study has reported a dramatic increase in sleep disorders of $111 \%$ following hip arthroscopy. ${ }^{18}$ However, there are limited studies investigating the incidence of sleep disturbances following arthroscopic knee surgery such as anterior cruciate ligament (ACL) reconstruction. Therefore, the purpose of this study was to evaluate the self-reported incidence of sleep disturbances, defined as $\leq 7$ hours of sleep per 24-hour period, in patients undergoing arthroscopic-assisted knee surgery. The hypothesis was that patients would report significantly decreased hours of sleep from preoperative to postoperative states.

\section{METHODS}

This study was approved following review from an institutional review board (institution and protocol number blinded for review). All patients who underwent arthroscopic knee surgery by a single board-certified orthopaedic surgeon (initials blinded for review) from September 2019 to December 2019 were prospectively enrolled. Demographics and surgical procedural data were collected on all patients including age, sex, body mass index (BMI), chronicity, time from injury to surgery, previous history of knee surgery, medical history (including osteoarthritis), social habits (smoking, tobacco and alcohol use), current medications, surgical procedure, tourniquet time and postoperative weight bearing status. All patients had general anaesthesia and prescription of the same narcotic (Oxycodone, $5 \mathrm{mg} \mathrm{\# 40}$ quantity, as needed; patients were also provided a self-disposal narcotic neutralisation bag) for postoperative analgesia. All patients started physical therapy on postoperative day 1 . During the first postoperative physical therapy session, knee circumference was measured at the level of the joint line in all patients and a side-to-side difference was recorded. This served as an objective baseline indicator of knee joint effusion.

Based on the extent of the surgical procedure, patients were divided posthoc into three groups for subanalysis: 'mild', 'moderate' and 'severe' knee surgery (table 1). This grouping was developed according to size of approach, anticipated postoperative pain and weight bearing status following the procedure. The rationale for this subdivision was to attempt to control for postoperative pain and disability associated with different surgical procedures (eg, meniscectomy vs ACL reconstruction) which may affect postoperative sleep metrics. Patients were excluded if they reported a current or prior history of insomnia or other sleep altering medical history (antidepressant medication, sleep apnoea), were less than 18 years of age, had a current history of osteoarthritis or underwent predominantly open knee surgical procedures (fracture open reduction internal fixation, tibial/femoral osteotomy, osteoarticular allograft transplant, meniscal allograft transplant, multiligament knee reconstruction). Complications that occurred in the first 6 weeks postoperatively were recorded.

\section{Sleep metrics}

Self-reported sleep metrics were collected from all included patients during the first 6 weeks following surgery. Preoperatively, patients were questioned regarding their baseline sleeping metrics, including the average number of hours of sleep per night and average number of awakenings during sleep per night. Patients were asked to keep a sleep diary of their sleeping habits and specific metrics during their postoperative recovery. Selfreported sleep metrics included the average number of hours of sleep per night, average number of awakenings during sleep per night because of their knee, perceived quality of sleep, average knee pain level at night during sleep and number of hours of physical therapy per week. In addition, data were collected on postoperative sleeping habits including the use of a knee brace, ice or ice machine, pillow underneath knee and body position (traditionally/preoperatively and during postoperative state). In conjunction with these sleep metrics, patients were surveyed to report their perceived causes of sleep disturbances and also their self-reported remedies that improved their perceived sleep disturbances. Surveys were completed at three different timepoints: weeks 1, 3 and 6 postoperatively. Missing data were reconciled via email of survey correspondents. Sleeping was classified as either 'disturbed': sleeping $\leq 7$ hours per 24-hour period or 'non-disturbed': sleeping $>7$ hours per 24 -hour period. ${ }^{12}$

\section{Statistical analysis}

To assess for normal distribution, histograms of outcome variables were generated prior to statistical analysis which demonstrated symmetrical bell curve distribution of sample data. Additionally, descriptive statistics determined no substantial skewness $(0.2-0.7)$ of mean values for outcome variables. To address the primary hypothesis, paired-sample $t$ tests were performed to compare hours of sleep from preinjury to postoperatively at weeks 1, 3 and 6. Ordinary least squares regression was used to test the association between the presence of a postoperative knee effusion and number of hours of sleep per week in the acute phase ( $<6$ weeks) following knee arthroscopy. Multiple linear regression modelling was performed to estimate the unadjusted and adjusted effect of postoperative knee effusion on predicting sleep metrics. In the multiple linear regression model, age, sex, BMI, surgery severity, tourniquet time, injury chronicity, postoperative weight bearing status, postoperative pain level, hours of physical therapy per week and previous history of knee surgery were entered as possible confounders. A sample size calculation was made to determine the required sample size necessary to detect a Pearson correlation magnitude of 0.3 , which is conventionally considered a "medium" strength correlation. Based on the assumption of 2-tailed testing and an alpha level of $0.05,82$ patients were sufficient to detect an effect size of $|\mathrm{r}|=0.3$ with $80 \%$ statistical power. Residual diagnostics were performed to

\begin{tabular}{|c|c|c|c|}
\hline $\begin{array}{l}\text { Severity of } \\
\text { surgery }\end{array}$ & $\begin{array}{l}\text { Sample size } \\
(\%)\end{array}$ & $\begin{array}{l}\text { Weight } \\
\text { bearing status }\end{array}$ & Knee surgical procedures \\
\hline \multirow[t]{2}{*}{ Moderate } & \multirow[t]{2}{*}{$41(49 \%)$} & WBAT & ACLR (with or without meniscal repair), bone grafting of previous ligament reconstruction tunnels \\
\hline & & NWB & Isolated meniscus repair, ACLR with meniscal radial or root repair \\
\hline
\end{tabular}




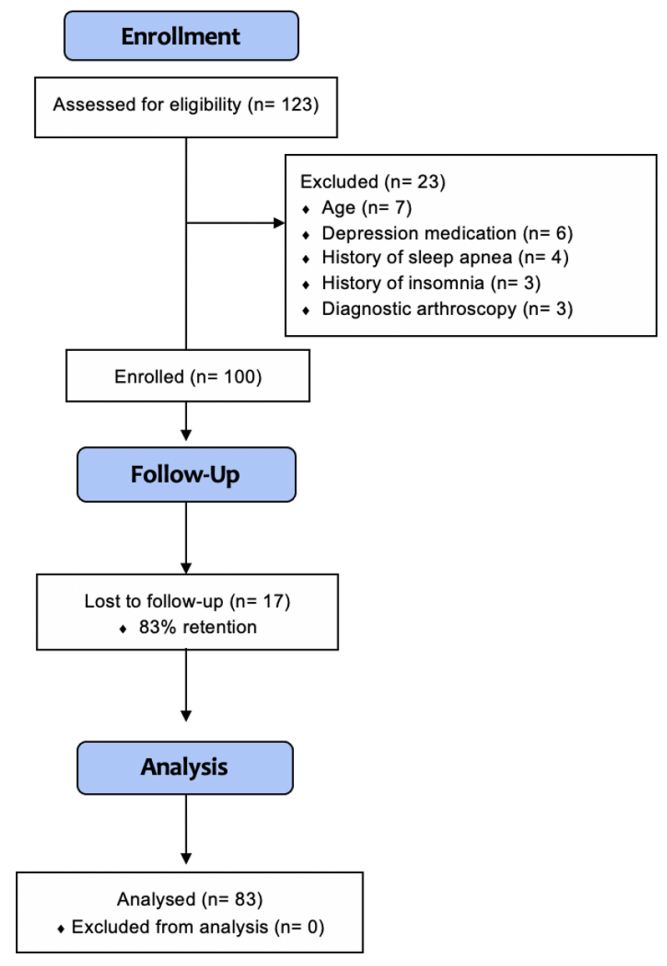

Figure 1 Flow chart of prospective enrolment of consecutive patients who underwent arthroscopic knee surgery during study period.

assess whether model assumptions were satisfactorily met. For the subanalysis on severity of surgery classification, a composite score was calculated to determine the overall mean number of hours of sleep postoperatively. A one-way analysis of variance was performed on mean number of hours of sleep postoperatively according to severity of surgical procedures ('mild', 'moderate', 'severe'). All data were analysed using SPSS V.25 (Chicago, Illinois, USA).

\section{RESULTS}

During the open enrolment period, 123 consecutive patients underwent arthroscopic knee surgery. Twenty-three patients were excluded at time of enrolment and 17 patients were lost to follow-up (83\% retention rate) during the 6-week data collection period; 83 patients ( 38 male, 45 female) were included in the final analysis (figure 1 ). The average age was $41.0 \pm 14.4$ years (range, 18-66 years). Fifty-five patients had chronic injuries ( $>12$ weeks from time of injury to surgery) and 28 patients had acute injuries ( $\leq 12$ weeks). The average overall tourniquet time was $40.6 \pm 28.0 \mathrm{~min}$ (range, $7-120 \mathrm{~min}$ ) and 55 patients were non-weight bearing in the first 6 weeks following surgery while the remaining 28 were permitted to weight bear as tolerated. The average time to discontinuation of pain medicine was $8.0 \pm 2.2$ days (range, $1-17$ days). There were no reported surgical complications in the first 6 weeks postoperatively; however, one patient (1.2\%) developed and was treated for atelectasis with mobilisation and incentive spirometry during the early postoperative period and resolved without sequelae. Patient demographics and clinical characteristics are presented in table 2 .

The average self-reported number of hours of sleep per night preoperatively was $8.0 \pm 0.9$ hours (range, 5-10), with $20 \%$ $(n=17)$ of patients reporting $\leq 7$ hours of regular sleep preoperatively. The incidence of self-reported sleep disturbances was
Table 2 Demographics and clinical characteristics for all included patients $(n=83)$

\begin{tabular}{lc}
\hline Demographic/clinical characteristic & Value \\
\hline Age (years) & $41.0 \pm 14.4$ \\
\hline Sex & \\
$\quad$ Male & $38 / 46 \%$ \\
$\quad$ Female & $45 / 54 \%$ \\
\hline Body mass index $\left(\mathrm{kg} / \mathrm{m}^{2}\right)^{*}$ & $26.6 \pm 5.8$ \\
\hline Tourniquet time $(\mathrm{min})^{*}$ & $40.6 \pm 28.0$ \\
Previous history of knee surgery & $43 / 52 \%$ \\
Current tobacco smoker & $1 / 1 \%$ \\
Current alcohol user & $36 / 43 \%$ \\
Postoperative complications (within first 6 weeks) & $1 / 1 \%$ \\
Knee joint effusion, postoperative day 1 $(\mathrm{cm})^{*}$ & $3.2 \pm 1.9$ \\
\hline Values reported as n/\%. & \\
*Data reported as mean \pm SD. &
\end{tabular}

$99 \%, 96 \%$ and $90 \%$ at postoperative weeks 1,3 and 6 , respectively. There were significant reductions in the average number of self-reported hours of sleep from preinjury to postoperative $(5.6 \pm 1.3,6.0 \pm 1.3$ and $6.2 \pm 1.2$ hours for postoperative weeks $1,3$ and 6 , respectively; $\mathrm{p}<0.001)$. There was a significant negative correlation between vknee joint circumference and the number of hours of sleep postoperatively $(R=-0.704 ; \mathrm{p}=0.001)$ (figure 2). There were no significant correlations identified between postoperative sleep and postoperative nighttime pain $(R=-0.121 ; \mathrm{p}=0.276)$ or hours of physical therapy $(R=-0.024$; $\mathrm{p}=0.829)$. No significant differences were found between severity of surgical procedures and mean number of hours of sleep postoperatively $(6.3 \pm 0.6,5.8 \pm 0.9$ and $5.9 \pm 1.1$ hours for 'mild', 'moderate' and 'severe' surgical procedures, respectively; $\mathrm{p}=0.147$ ). Multivariable regression analysis revealed that other surgical variables were not significant independent predictors of postoperative sleep (table 3). Descriptive data of postoperative sleep metrics are reported in table 4.

The most common patient-reported causes of sleep disturbances were: knee 'warmth' $(\mathrm{n}=80,96 \%)$ at night, wearing a knee brace to sleep $(n=75,90 \%)$ and not sleeping in a normal body position compared with presurgery $(n=72,87 \%)$. The most common patient-reported therapeutic factors for improving

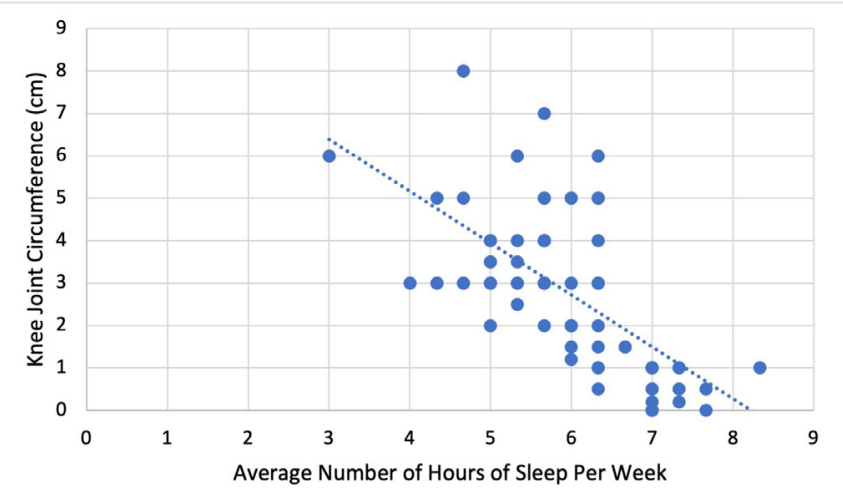

Figure 2 Scatterplot of unadjusted linear regression relationship between postoperative knee effusion and postoperative sleep duration. There was a significant negative correlation between the postoperative day 1 knee joint circumference and average number of hours of sleep per week during the acute phase ( 6 weeks) following arthroscopic knee surgery $(R=-0.704 ; p=0.001)$. 
Original research

Table 3 Multivariable linear regression model for surgical variables and postoperative hours of sleep

\begin{tabular}{lrllll}
\hline & Beta* $^{*}$ & $95 \% \mathrm{Cl}$ & $\mathrm{SE}$ & $\boldsymbol{t}$ value & P valuet \\
\hline (Intercept) & 7.579 & 6.404 to 8.754 & 0.590 & 12.85 & 0.001 \\
\hline Knee effusion & -0.373 & -0.467 to -0.280 & 0.047 & -7.96 & 0.001 \\
Age & -0.004 & -0.017 to 0.008 & 0.006 & -0.66 & 0.509 \\
\hline Sex & -0.213 & -0.549 to 0.124 & 0.169 & -1.26 & 0.212 \\
$\begin{array}{l}\text { Body mass } \\
\text { index }\end{array}$ & 0.010 & -0.018 to 0.038 & 0.014 & 0.72 & 0.474 \\
$\begin{array}{l}\text { Chronicity of } \\
\text { injury }\end{array}$ & -0.050 & -0.438 to 0.338 & 0.195 & -0.25 & 0.798 \\
$\begin{array}{l}\text { Severity of } \\
\text { surgery }\end{array}$ & -0.261 & -0.660 to 0.139 & 0.201 & -1.30 & 0.198 \\
$\begin{array}{l}\text { Tourniquet } \\
\text { time }\end{array}$ & -0.003 & -0.011 to 0.005 & 0.004 & -0.82 & 0.412 \\
$\begin{array}{l}\text { Weight } \\
\text { bearing status }\end{array}$ & -0.061 & -0.569 to 0.447 & 0.255 & -0.24 & 0.811 \\
$\begin{array}{l}\text { Previous } \\
\text { history of } \\
\text { knee surgery }\end{array}$ & 0.299 & -0.084 to 0.683 & 0.192 & 1.55 & 0.124 \\
\hline & & & & & \\
\hline
\end{tabular}

Statistically significant variables are provided in bold.

*Beta values are the expected change in sleep given a 1-unit decrease in that covariate, holding all other variables constant.

†Statistical significance considered $p<0.05$.

postoperative sleep metrics were: placing a pillow underneath the knee during sleep $(n=82,99 \%)$, removal of knee brace during sleep $(n=79,95 \%)$ and performing ice, compression and elevation (ICE) during sleep $(n=69,83 \%)$.

\section{DISCUSSION}

The main finding of this study was that patients reported acute sleep disturbances following arthroscopic knee surgery without correction of baseline sleep patterns by 6 weeks postoperatively. A significant negative correlation was identified between the postoperative sleep hours and a postoperative knee effusion. Additionally, pain level and hours of physical therapy were not significantly associated with sleep metrics per patient-reported data. The primary self-reported causes of acute sleep disturbances were knee 'warmth' (swelling), wearing a knee brace and not sleeping in a normal body position. The most frequent selfreported therapeutic factors for improving sleep were placing a pillow underneath the knee, removal of knee brace and using the ICE principal (ice, compression, elevation).

The overall incidence of self-reported preoperative sleep disturbances ( $\leq 7$ hours) was $20 \%$ which increased to $99 \%$, $96 \%$ and $90 \%$ at postoperative weeks 1, 3 and 6, respectively, following arthroscopic knee surgery. The average number of hours slept was significantly reduced at 1,3 and 6 weeks postoperatively compared with the preoperative state. Previous studies report similar sleep metrics following knee and hip arthroplasty, with corrections of sleep to preoperative baseline at 10 months postoperatively. ${ }^{16} 17$ Additionally, a recent study reported a $111 \%$ increase in sleep disorders following hip arthroscopy. ${ }^{18}$ In contrast, Hudson et al ${ }^{19}$ reported that ambulatory surgery and anaesthesia had minimal effect on sleep parameters 1-3 days after orthopaedic surgery. However, their study purpose was to measure changes in perioperative sleep parameters, specifically evaluating presence of sleep apnoea; thus, these results are not directly comparable to the present study. Also, in the current study, nighttime pain was not associated with any sleep metrics in the first 6 weeks following arthroscopic knee surgery. This finding was supported by a randomised controlled trial that failed to improve postoperative sleep following lower extremity surgery with the use of prescription medication (Gabapentin) for pain control. ${ }^{20}$ Considering the high incidence of acute sleep disturbances demonstrated in this study of consecutive adult patients undergoing arthroscopic knee surgery, it is recommended that further research should focus on developing sleep intervention therapy for this specific patient-population. ${ }^{15} 21-24$

Table 4 Sleep metrics following knee surgery reported at preoperative and postoperative time points

\begin{tabular}{|c|c|c|c|c|}
\hline Sleep-related metric & Preoperative value & Week 1 value & Week 3 value & Week 6 value \\
\hline Nightly sleep duration (hours)* & $8.0 \pm 0.9$ & $5.6 \pm 1.3$ & $6.0 \pm 1.3$ & $6.2 \pm 1.2$ \\
\hline \multicolumn{5}{|l|}{ Nightly awakenings } \\
\hline 0 & $66 / 80 \%$ & $0 / 0 \%$ & $0 / 0 \%$ & $4 / 5 \%$ \\
\hline 1 & $7 / 8 \%$ & $14 / 17 \%$ & $27 / 33 \%$ & $39 / 47 \%$ \\
\hline 2 & $7 / 8 \%$ & $11 / 13 \%$ & $16 / 19 \%$ & $14 / 17 \%$ \\
\hline 3 & $2 / 2 \%$ & $16 / 19 \%$ & $10 / 12 \%$ & $11 / 13 \%$ \\
\hline $4+$ & $1 / 1 \%$ & $42 / 51 \%$ & $30 / 36 \%$ & $15 / 18 \%$ \\
\hline \multicolumn{5}{|l|}{ Sleep quality } \\
\hline $1=$ very poor & & $17 / 20 \%$ & $12 / 14 \%$ & $6 / 7 \%$ \\
\hline $2=p o o r$ & & $34 / 41 \%$ & $27 / 33 \%$ & $14 / 17 \%$ \\
\hline $3=$ average & & $21 / 25 \%$ & $24 / 29 \%$ & $28 / 34 \%$ \\
\hline $4=$ good & & $10 / 12 \%$ & $16 / 19 \%$ & $27 / 33 \%$ \\
\hline $5=e x c e l l e n t$ & NR & $1 / 1 \%$ & $4 / 5 \%$ & $8 / 10 \%$ \\
\hline \multicolumn{5}{|l|}{ Nighttime pain level } \\
\hline $0=$ none & & $14 / 17 \%$ & $33 / 40 \%$ & $54 / 65 \%$ \\
\hline $1=$ mild & & $49 / 59 \%$ & $41 / 49 \%$ & $26 / 31 \%$ \\
\hline $2=$ moderate & & $18 / 22 \%$ & $9 / 11 \%$ & $3 / 4 \%$ \\
\hline $3=$ severe & NR & $2 / 22 \%$ & $0 / 0 \%$ & $0 / 0 \%$ \\
\hline Weekly hours of physical therapy* & NR & $9.0 \pm 6.2$ & $8.5 \pm 5.2$ & $9.2 \pm 5.0$ \\
\hline Sleep disturbance & $17 / 20 \%$ & $82 / 99 \%$ & $81 / 96 \%$ & $75 / 90 \%$ \\
\hline
\end{tabular}

Values reported as $\mathrm{n} / \%$.

${ }^{*}$ Data reported as mean $\pm \mathrm{SD}$.

NR, not reported. 
A significant negative correlation was identified between postoperative sleep hours and a postoperative knee effusion, as patients with increased knee swelling had decreased self-reported hours of sleep per night. This was also supported by our finding of selfreported knee 'warmth' as a contributing factor that negatively affected sleep. A potential theory to describe why patients report sleep disturbances following orthopaedic surgery relates to an increase in joint inflammation. C reactive protein, interleukin 6 and tumour necrosis factor are three commonly measured inflammatory markers that are increased in patients with sleep disorders. ${ }^{1425} 26$ Similarly, studies have reported increased levels of the same inflammatory markers following orthopaedic surgery and high elevations of these markers may negatively impact musculoskeletal healing. ${ }^{1327}$ Thus, in theory, the larger the healing response associated with the surgery and recovery, the greater the potential sleep disturbance. However, additional research is needed to further evaluate the role of sleep disturbances on the impact of elevated inflammatory markers and musculoskeletal tissue healing.

There was no significant correlation found between postoperative sleep and number of hours performing postoperative physical therapy. There are many environmental and behavioural factors that may influence sleep, including screen time (eg, TV, phone, computer), psychological stress and anxiety and caffeine use-all of which were not controlled for in the current study. ${ }^{28-30}$ However, the current study attempted to account for physical inactivity which is also a commonly reported influential factor affecting number of hours of sleep per night. ${ }^{7}$ Previous studies have demonstrated that the quantity of sleep the quality of sleep are important, a combined term referred to in the sleep literature as 'sleeping efficiency'. ${ }^{31}$ Future studies ought to examine sleeping efficiency and evaluate potential associations with physical activity levels in patients following orthopaedic knee surgery. ${ }^{23}$

In 2021, a reported 6.6 million individuals will undergo orthopaedic surgery in the USA alone. ${ }^{32}$ Considering the current $90 \%$ incidence of self-reported sleep disturbances in the current study, there is a potential for 5.9 million people per year to experience negative sleep metrics as a result of orthopaedic surgery in the USA alone. Although this scenario is hypothetical, it can offer encouragement to scientists, clinicians as well as members of industry to increase research efforts in reducing postoperative sleep disturbances following orthopaedic-related surgery. Results from the current study suggest improving strategies focused on reducing knee swelling, including preoperative and intraoperative care, as well as postoperative management.

Although there is limited clinical evidence to indicate that sleep disturbances affect overall recovery or functional impairment following orthopaedic surgery, insufficient sleep is a major reported health concern with associations to obesity, diabetes, cardiovascular disease and mental health. ${ }^{4} 101133-35$ Because of these detrimental consequences, allied healthcare professionals involved in the care of orthopaedic surgical patients should take the initiative to improve this metric in healthcare systems. Based on the available evidence and results of the current study, a multidisciplinary team approach is recommended to help educate and counsel patients regarding the potential for acute sleep disturbances following arthroscopic knee surgery (such as ACL reconstruction). The team member at the centre of this team approach is the orthopaedic surgeon. Perioperative team members include nurses, physician assistants and anaesthesiologists, while postoperative team members include physical therapists, athletic trainers and sport psychologists.

This study is not without limitations. First, due to the nature of studying uncontrolled sleep settings, there was an inability to control for potential confounding variables that may have also affected sleep such as environmental and behavioural factors. Second, data are indicated based on patient-reported sleep metrics which are subjective and lack clinical validation. Future studies should consider implementing wearable technology devices (eg, smart watch, actigraphy) for a more accurate and objective assessment of sleep quantity and quality postoperatively. ${ }^{36}$ Third, daytime sleeping/napping was unable to be controlled for which may have influence over nighttime sleep data. Finally, long-term sleep corrections or associations of sleep disturbances with surgical outcomes were not able to be assessed due to the study design. Future studies should examine the long-term consequences of sleep disturbances following arthroscopic surgery and evaluate therapeutic agents that may improve sleeping metrics.

\section{CONCLUSION}

Sleep disturbances were commonly reported in patients following arthroscopic knee surgery without correction of sleep metrics by 6 weeks postoperatively. The majority of sleep disturbances in this cohort correlated with an increased knee effusion. A multidisciplinary team approach is recommended to counsel patients regarding the potential for and problems with acute sleep disturbances following arthroscopic knee surgery.

\section{Twitter Jill Monson @jillMonsonPT}

Contributors NND: primary writing contribution, data collection, data analysis, idea generation, formatting, patient consent. RD: primary writing contribution, data collection, idea generation. LE: significant writing contribution, data collector, data analysis. CML: significant writing contribution, data collector, data analysis. JM: writing contribution, data collector, data analysis. RL: critical edits, manuscript writing and idea generation.

Funding The authors have not declared a specific grant for this research from any funding agency in the public, commercial or not-for-profit sectors.

Competing interests None declared.

Patient consent for publication Not required.

Ethics approval This was study was approved by an external IRB committee.

Provenance and peer review Not commissioned; externally peer reviewed.

Data availability statement All data relevant to the study are included in the article or uploaded as supplementary information. All data relevant to the study are included in the article or uploaded as supplementary information.

\section{ORCID iDs}

Nicholas N DePhillipo http://orcid.org/0000-0001-8946-4028

Jill Monson http://orcid.org/0000-0001-8584-8614

\section{REFERENCES}

1 Austin L, Pepe M, Tucker B, et al. Sleep disturbance associated with rotator cuff tear: correction with arthroscopic rotator cuff repair. Am J Sports Med 2015;43:1455-9.

2 Horneff JG, Tjoumakaris F, Wowkanech C, et al. Long-Term correction in sleep disturbance is sustained after arthroscopic rotator cuff repair. Am J Sports Med 2017;45:1670-5.

3 Jung JH, Seok H, Choi SJ, et al. The association between osteoarthritis and sleep duration in Koreans: a nationwide cross-sectional observational study. Clin Rheumatol 2018;37:1653-9

4 Cappuccio FP, Miller MA. Sleep and Cardio-Metabolic disease. Curr Cardiol Rep 2017; 19:110.

5 Ford ES, Wheaton AG, Chapman DP, et al. Associations between self-reported sleep duration and sleeping disorder with concentrations of fasting and 2-h glucose, insulin, and glycosylated hemoglobin among adults without diagnosed diabetes. J Diabetes 2014;6:338-50.

6 Kruisbrink M, Robertson W, Ji C, et al. Association of sleep duration and quality with blood lipids: a systematic review and meta-analysis of prospective studies. BMJ Open 2017;7:e018585.

7 Xiao Q, Keadle SK, Hollenbeck AR, et al. Sleep duration and total and cause-specific mortality in a large US cohort: interrelationships with physical activity, sedentary behavior, and body mass index. Am J Epidemiol 2014;180:997-1006.

8 Xie L, Kang H, Xu Q, et al. Sleep drives metabolite clearance from the adult brain. Science 2013;342:373-7.

9 Cespedes Feliciano EM, Quante M, Rifas-Shiman SL, et al. Objective sleep characteristics and cardiometabolic health in young adolescents. Pediatrics 2018;142. doi:10.1542/peds.2017-4085. [Epub ahead of print: 1506 2018]. 
10 Ford ES, Li C, Wheaton AG, et al. Sleep duration and body mass index and waist circumference among U.S. adults. Obesity 2014;22:598-607.

11 Reardon CL, Hainline B, Aron CM, et al. Mental health in elite athletes: international Olympic Committee consensus statement (2019). Br J Sports Med 2019;53:667-99.

12 Watson NF, Badr MS, Belenky G, et al. Recommended amount of sleep for a healthy adult: a joint consensus statement of the American Academy of sleep medicine and sleep research Society. Sleep 2015;38:843-4.

13 Neumaier M, Braun KF, Sandmann G, et al. C-Reactive protein in orthopaedic surgery. Acta Chir Orthop Traumatol Cech 2015;82:327-31.

14 Tanaka T, Narazaki M, Kishimoto T. II-6 in inflammation, immunity, and disease. Cold Spring Harb Perspect Biol 2014;6:a016295.

15 Austin L. Editorial commentary: does orthopedic disease lead to sleep disturbance, and how can we improve sleep quality following surgery? Arthroscopy 2019;35:470.

16 Chen AF, Orozco FR, Austin LS, et al. Prospective evaluation of sleep disturbances after total knee arthroplasty. J Arthroplasty 2016:31:330-2.

17 Manning BT, Kearns SM, Bohl DD, et al. Prospective assessment of sleep quality before and after primary total joint replacement. Orthopedics 2017;40:e636-40.

18 Rhon DI, Greenlee TA, Marchant BG, et al. Comorbidities in the first 2 years after arthroscopic hip surgery: substantial increases in mental health disorders, chronic pain, substance abuse and cardiometabolic conditions. Br J Sports Med 2019;53:547-53.

19 Hudson AJ, Walter RJ, Flynn J, et al. Ambulatory surgery has minimal impact on sleep parameters: a prospective observational trial. J Clin Sleep Med 2018;14:593-602.

20 Eloy JD, Anthony C, Amin S, et al. Gabapentin does not appear to improve postoperative pain and sleep patterns in patients who concomitantly receive regional anesthesia for lower extremity orthopedic surgery: a randomized control trial. Pain Res Manag 2017;2017:1-5

21 Bonnar D, Bartel K, Kakoschke N, et al. Sleep interventions designed to improve athletic performance and recovery: a systematic review of current approaches. Sports Med 2018;48:683-703.

22 Su X, Wang D-X. Improve postoperative sleep: what can we do? Curr Opin Anaesthesiol 2018;31:83-8.
23 Kroshus E, Wagner J, Wyrick D, et al. Wake up call for collegiate athlete sleep: narrative review and consensus recommendations from the NCAA Interassociation Task force on sleep and wellness. Br J Sports Med 2019;53:731-6.

24 Wong EM-L, Chair S-Y, Leung DYP, et al. Can a brief educational intervention improve sleep and anxiety outcomes for emergency orthopaedic surgical patients? Contemp Nurse 2014:47:132-43.

25 Bradley JR. Tnf-Mediated inflammatory disease. J Pathol 2008;214:149-60.

26 Richardson MR, Churilla JR, Duration S. Sleep duration and C-reactive protein in US adults. South Med J 2017;110:314-7.

27 Timmen $\mathrm{M}$, Hidding $\mathrm{H}$, Wieskötter $\mathrm{B}$, et al. Influence of antiTNF-alpha antibody treatment on fracture healing under chronic inflammation. BMC Musculoskelet Disord 2014;15:184.

28 Chang A-M, Aeschbach D, Duffy JF, et al. Evening use of light-emitting eReaders negatively affects sleep, circadian timing, and next-morning alertness. Proc Natl Acad Sci U SA 2015;112:1232-7.

29 Halson SL. Stealing sleep: is sport or Society to blame? Br J Sports Med 2016;50:381.

30 Wheaton AG, Olsen Emily O'Malley, Miller GF, et al. Sleep Duration and Injury-Related Risk Behaviors Among High School Students--United States, 2007-2013. MMWR Morb Mortal Wkly Rep 2016;65:337-41.

31 Shrivastava D, Jung S, Saadat M, et al. How to interpret the results of a sleep study. J Community Hosp Intern Med Perspect 2014;4:24983.

32 United States bone and joint initiative: the burden of musculoskeletal diseases in the United States, 3rd edn. Rosemont, IL: United States Bone and Joint Initiative, 2014. Available: http://bmus.latticegroup.com [Accessed 27 Mar 2020].

33 Hart CN, Cairns A, Jelalian E. Sleep and obesity in children and adolescents. Pediatr Clin North Am 2011:58:715-33.

34 Kamath MG, Prakash J, Dash S, et al. Is there an Association Between Self-Reported Sleep Duration, Body Mass Index and Waist-Hip Ratio in Young Adults? A CrossSectional Pilot Study. J Clin Diagn Res 2014;8:BC05-7.

35 Reardon CL, Hainline B, Aron CM, et al. Infographic. Sleep disorders in athletes. $\mathrm{Br}$ J Sports Med 2020;54:188-9.

36 Martin JL, Hakim AD. Wrist actigraphy. Chest 2011;139:1514-27. 